Annals of Pure and Applied Mathematics

Vol. 14, No. 1, 2017, 143-152

ISSN: 2279-087X (P), 2279-0888(online)

Published on 31 July 2017

Annals of

www.researchmathsci.org

DOI: http://dx.doi.org/10.22457/apam.v14n1a17

Pure and Applied

Mathematics

\title{
Edge Roman Star Domination Number on Graphs
}

Angshu Kumar Sinha ${ }^{1}$, Akul Rana ${ }^{2}$ and Anita Pal ${ }^{3}$

${ }^{1}$ Department of Mathematics, NSHM Knowledge Campus

Durgapur -713212, INDIA. e-mail: angshusinha20@ gmail.com

${ }^{2}$ Department of Mathematics, Narajole Raj College

Narajole, Paschim Medinipur- 721211, INDIA. e-mail: arnrc79@gmail.com

${ }^{3}$ Department of Mathematics, National Institute of Technology Durgapur

Durgapur-713209, INDIA

Corresponding author. e-mail: anita.buie@gmail.com

Received 12 June 2017; accepted 21 July 2017

Abstract. In this paper, we introduce a new variation of edge Roman domination, called edge Roman star domination. An edge Roman star domination function of a graph $G=(V, E)$ is a function $f: E \rightarrow\{0,1,2\}$ such that (i) sum of the weight of the edges incident on every vertex $v$ is at least 1 , and (ii) every edge $e$ with $f(e)=0$ is adjacent to at least one edge $e^{\prime}$ with $f\left(e^{\prime}\right)=2$. The weight of an edge Roman star domination function is the value $f(E)=\sum_{e \in E} f(e)$. The minimum of the values of an edge Roman star dominating function $f$ on a graph $G$, is called an edge Roman star domination number of $G$ and is denoted by $\gamma_{R S}^{\prime}(G)$. Here, we obtained the exact values of the edge Roman star domination number of paths, cycles, star graphs, and binary trees . Also, some bounds of $\gamma_{R S}^{\prime}(G)$ is presented for general graphs.

Keywords: Domination, Roman domination, edge Roman domination, edge Roman star domination

AMS Mathematics Subject Classification (2010): 05C69, 05 C78

\section{Introduction}

The mathematical study of domination in graphs began around 1960. In 1962, Oystein Ore first defined the domination number of a graph in his book 'The theory of Graphs' [14]. A decade later, Cockayne and Hedetiemi first used the notation $\gamma(G)$ for the domination number of a graph $G$ [8].

Definition 1. A set $S \subseteq V$ is said to be a dominating set of $G$, if every vertex in $V \backslash S$ is adjacent to some vertex in $S$. The minimum cardinality of such a set is called the domination number of $G$ and is denoted by $\gamma(G)$. 
Angshu Kumar Sinha, Akul Rana and Anita Pal

The concept of edge domination was introduced by Mitchell and Hedetniemi [13]. Domination and its variations have been extensively studied in the literature, see [1, $6,11,12,17,21,22,25,26]$.

Definition 2. A set $F$ of edges in a graph $G$ is an edge dominating set if every edge in $E \backslash F$ is adjacent to at least one edge in $F$. The minimum number of edges in such a set is called the edge domination number of $G$ and is denoted by $\gamma^{\prime}(G)$.

One of the most important variants of domination is the Roman domination of graphs which has both historical and mathematical implication. In 4 th century A.D., Constantine the Great (Emperor of Rome) decreed that any city without a legion stationed to secure it must adjacent another city having two stationed legions. Motivated by an article 'Defend the Roman Empire' by Ian Stewart [24], the definition of the Roman dominating function was given implicitly by Revelle and Rosing [16].

Roman Domination and its variations have been extensively studied in the literature, see $[2,5,7,9,23]$.

Definition 3. A function $f: E \rightarrow\{0,1,2\}$ is defined as a Roman dominating function on a graph $G=(V, E)$ if the function $f$ satisfies the condition that every vertex $u$ for which $f(u)=0$ has at least one neighboring vertex $v$ with $f(v)=2$. The weight of a Roman dominating function is the value $f(V)=\sum_{v \in V} f(v)$. The Roman domination number of $G$ is the minimum weight of a Roman dominating function and is denoted by $\gamma_{R}(G)$.

The concept of edge Roman dominating function of the graphs was defined in [15]. The edge Roman domination function have been widely discussed in the literature, please see $[5,7]$.

Definition 4. A function $f: E \rightarrow\{0,1,2\}$ satisfying the condition that every edge $e$ for which $f(e)=0$ is incident to at least one edge $e^{\prime}$ for which $f\left(e^{\prime}\right)=2$ is called an edge Roman dominating function of the graph. The edge Roman domination number, denoted by $\gamma_{R}^{\prime}(G)$, equals the minimum weight of an edge Roman domination function of G.

A signed Roman dominating function is a variation of Roman dominating function and was introduced by Abdollahzadeh Ahangar et al [3]. Several authors has been studied signed Roman dominating function, see the literature [18, 19, 20]. Again the edge variation of signed Roman domination was first studied by Abdollahzadeh Ahangar et al [4].

Definition 5. A function $f: E \rightarrow\{-1,1,2\}$ is said to be a signed Roman star edge dominating function, if $f$ satisfies the conditions, $(i) \sum_{e \in E(v)} f(e) \geq 1$ for every $v \in V$ and (ii) for every edge $e$ with $f(e)=-1$ is adjacent to at least one edge $e^{\prime}$ with $f\left(e^{\prime}\right)=2$. The weight of a signed Roman star edge dominating function $f$ on $G$ is 
Edge Roman Star Domination Number on Graphs

$w(f)=\sum_{e \in E} f(e)$. The minimum of weights taken over all the signed Roman star edge dominating functions $f$ on $G$, is called the signed Roman star edge domination number of $G$, denoted by $\gamma_{\text {srs }}^{\prime}(G)$.

In this paper, we initiate the study of an edge Roman star domination function(ERSDF, for short) which is an extension of an edge Roman domination function. Here, we add one extra condition that the sum of the weight of edges at each vertex is at least one.

Therefore, edge Roman star domination (ERSD, for short) is one of the variations of the edge Roman domination, which includes a restriction that the sum of the weight of the edges incident at each vertex is atleast one. So for a graph $G$, edge Roman star domination function of the graphs $G$ implies the edge Roman domination function of that graphs, but the converse may be true. The edge Roman star domination function is defined as follows.

Definition 6. A function $f: E \rightarrow\{0,1,2\}$ is said to be an edge Roman star domination function, if $f$ satisfies the conditions, ( $i)$ sum of the weight of the edges incident on every vertex $v$ is at least 1 , and (ii) every edge $e$ with $f(e)=0$ is adjacent to at least one edge $e^{\prime}$ with $f\left(e^{\prime}\right)=2$. The weight of a ERSDF $f$ on $G$ is $f(E)=\sum_{e \in E} f(e)$. The minimum of weights taken over all the edge Roman star dominating functions $f$ on $G$, is called the edge Roman star domination number of $G$, denoted by $\gamma_{R S}^{\prime}(G)$.

\subsection{Preliminary and notation}

Through out the paper, the graph $G$ is a simple, finite and undirected graph with vertex set $V$ and edge set $E$. The order $|V|$ of $G$ is denoted by $n$ and size $|E|$ of $G$ is denoted by $m$.

A cycle of $n$ vertices is denoted by $C_{n}$, while a path of $m$ edges is denoted by $P_{m}$. A star is a complete bipartite graph of the form $K_{1, n}$. A vertex of degree one is called a pendant vertex and its neighbor is called a support vertex. The edge between a pendant vertex and a support vertex is called pendant edge. Let $P$ be the set of pendant edges. Let $f$ be an edge Roman star dominating function in $G$. The edge set is partitioned into three sets, namely, $E_{0}=\{e \in E: f(e)=0\}, E_{1}=\{e \in E: f(e)=1\}$, $E_{2}=\{e \in E: f(e)=2\}$ which determines $f$ as a ERSDF.

Let $E_{2}\left(v_{i}\right), E_{1}\left(v_{i}\right)$ and $E_{0}\left(v_{i}\right)$ are the set of edges with weight $2,1,0$ respectively incident on the vertex $v_{i}$.

A matching in a graph $G$ is a subset $M$ of edges of $E$ such that no two edges in $M$ are adjacent. A matching $M$ is maximal if for every $e \in E \backslash M, M \cup\{e\}$ is not 


\section{Angshu Kumar Sinha, Akul Rana and Anita Pal}

a matching. A maximum matching in a graph $G$ is a maximal matching containing the largest number of edges. A matching $M$ in $G$ is called a perfect matching if every vertex of $G$ is incident to some edge in $M$. Every Perfect matching is a maximum matching but the converse is not necessarily true. The number of edges of a maximum matching in a graph $G$ is called the matching number of the graph $G$.

A binary tree is defined as a tree in which there is exactly one vertex (root) of degree two and each of the remaining vertices is of the degree either one or three. If every internal vertex has two children, then the tree is called complete binary tree.

\section{Important results}

Let $f$ be a $E R S D F$ in a graph $G$. Observe that, every edge in $E_{0}$ is dominated by an edge in $E_{2}$.

It is easy to verify that $\gamma_{R S}^{\prime}(G)=\left|E_{1}\right|+2\left|E_{2}\right|$. Also, $E_{1} \cup E_{2}$ is an edge dominating set of $G$.

Lemma 1. Let $K_{1, n}$ be a star graph. Then $\gamma_{R S}^{\prime}\left(K_{1, n}\right)=n$.

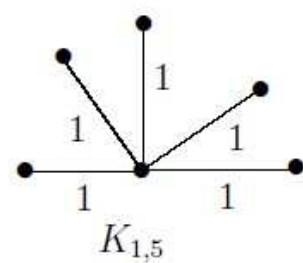

Figure 1: Star graph

Proof: Let $K_{1, n}$ be a star graph with vertex set $\left\{v_{0}, v_{1}, \ldots, v_{n}\right\}$ and $\left\{\left(v_{0}, v_{i}\right): i=1,2,3, \ldots, n\right.$. $\}$ be the set of pendant edges. If the weight of each edge is 0 , then clearly, $f$ is not a $E R S D F$. Again, if the weight of each edge is 2, then sum of weight of edges is not minimum. Here, the number of pendant edges is $n$. Thus, $\gamma_{R S}^{\prime}\left(K_{1, n}\right)=n$.

From the above lemma, it is obvious that if $f$ is a $E R S D F$, then the weight of each pendant edge of a graph $G$ is always 1 .

Lemma 2. For a path $P_{m}, \gamma_{R S}^{\prime}\left(P_{m}\right)= \begin{cases}m, & m \leq 4 \\ 2\left\lfloor\frac{m-2}{2}\right\rfloor+2, & \text { mis odd } \\ m-1, & \text { m is even }\end{cases}$

Proof: Let $P_{m}$ be the path of length $m$. For $m \leq 4$, the proof is obvious.

Let $E^{\prime}=E \backslash T^{\prime}$, where $T^{\prime}$ is the terminal edges of $P_{m}$. 


\section{Edge Roman Star Domination Number on Graphs}

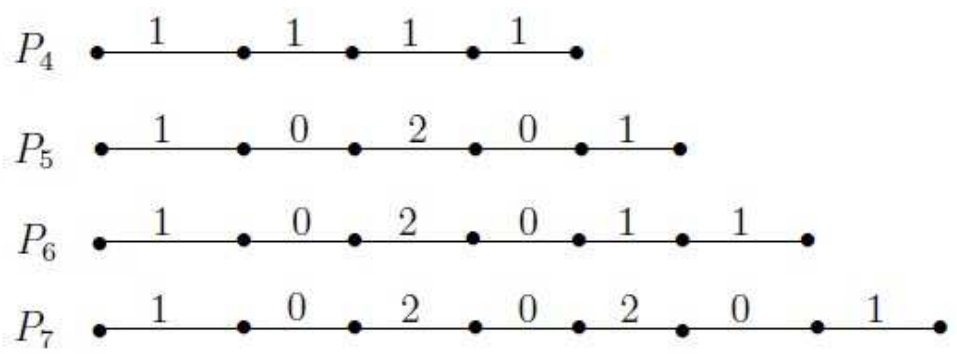

Figure 2: Paths

Let $M$ be a matching of the set of edges $E^{\prime}$.

If $m$ is odd, then the assignment of the weight of the edges for the path $P_{m}$ is as follows.

$$
\begin{aligned}
f(e) & =0, \quad e \in M \\
& =2, \quad e \in E^{\prime} \backslash M \\
& =1, \quad e \in E \backslash E^{\prime}
\end{aligned}
$$

Here, each edge with weight 2 has two neighboring edges with weight 0 . Therefore, the number of edges with weight 2 is one less than the number edges with weight 0 . The number of edges in matching $M$ is $\left\lceil\frac{m-2}{2}\right\rceil$.

Thus, $\left|E_{0}\right|=\left\lceil\frac{m-2}{2}\right\rceil,\left|E_{2}\right|=\left\lfloor\frac{m-2}{2}\right\rfloor$ and $\left|E_{1}\right|=2$. In this case the sum of the weight of all edges at each vertex of the path $P_{m}$ is either 2 or 1 . Hence $f$ is a ERSDF.

Hence, if $m$ is odd, then

$$
\gamma_{R S}^{\prime}\left(P_{m}\right)=2\left|E_{2}\right|+0 .\left|E_{0}\right|+\left|E_{1}\right|=2 .\left\lfloor\frac{m-2}{2}\right\rfloor+0 \cdot\left\lceil\frac{m-2}{2}\right\rceil+2 .=2 \cdot\left\lfloor\frac{m-2}{2}\right\rfloor+2 .
$$

Now if $m$ is even, assignment of the edges of the path $P_{m}$ as follows.

$$
\begin{aligned}
f(e) & =0, \quad e \in M \\
& =2, \quad e \in E^{\prime} \backslash M \\
& =1, \quad e \in E \backslash E^{\prime}
\end{aligned}
$$

Here, the number of edges in $M$ is $\frac{m-2}{2}$. Since the weight of each edge in $M$ is 0 , therefore $\left|E_{0}\right|=\frac{m-2}{2}$. Again, the edge with weight 2 is bounded by the two edges with weight 0 . Thus, the number of edges with weight 2 is one less than the number of edges with weight 0 , therefore $\left|E_{2}\right|=\frac{m-2}{2}-1$. Here, the total number edges with weight 1 are three (including the terminal edges). In this case the sum of the weight 
Angshu Kumar Sinha, Akul Rana and Anita Pal

of all edges at each vertex of the path $P_{m}$ is either 2 or 1 and hence $f$ is a ERSDF. Thus, $\gamma_{R S}^{\prime}\left(P_{m}\right)=2\left(\frac{m-2}{2}-1\right)+0 .\left(\frac{m-2}{2}\right)+3=m-1$.

Lemma 3. For a even cycle $C_{n}$, $\gamma_{R S}^{\prime}\left(C_{n}\right)= \begin{cases}2\left\lfloor\frac{n}{2}\right\rfloor-1, & \text { matching number is odd } \\ \frac{3}{2}\left\lfloor\frac{n}{2}\right\rfloor, & \text { matching number is even }\end{cases}$ And for a odd cycle $C_{n}, \gamma_{R S}^{\prime}\left(C_{n}\right)=\left\{\begin{array}{ll}3, & n=3 \\ 2\left\lfloor\frac{n}{2}\right\rfloor, & n>3\end{array}\right.$.

Proof: Let $C_{n}$ be a cycle of order $n$.

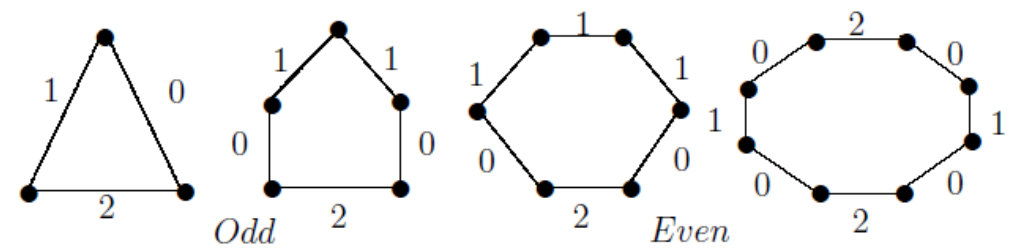

Figure 3: Cycles

The number of edges in the matching $M$ is $\left\lfloor\frac{n}{2}\right\rfloor$, i.e., matching number is $\left\lfloor\frac{n}{2}\right\rfloor$ . If $n$ is even, then $M$ is a perfect matching. If $n$ is odd, then $M$ is a maximum matching.

If the cycle is even and matching number is odd, then first assign weight 0 to the edges in $M$. Remaining $\left\lfloor\frac{n}{2}\right\rfloor-1$ number of edges are assigned by 2 and one edge is assigned by weight 1 . Here, the sum of the weight of all the edges at every vertex is either 2 or 1 and the edge of weight 0 has a neighboring edge with weight 2 . Hence, $f$ is a $E R S D F$.

Thus, $\gamma_{R S}^{\prime}\left(C_{n}\right)=2\left|E_{2}\right|+\left|E_{1}\right|=2\left(\left\lfloor\frac{n}{2}\right\rfloor-1\right)+1=2\left\lfloor\frac{n}{2}\right\rfloor-1$

If the cycle is even and matching number is even, then assign a weight 0 to the edges in $M$. Remaining $\frac{1}{2}\left\lfloor\frac{n}{2}\right\rfloor$ number of edges are assigned by 2 whereas other $\frac{1}{2}\left\lfloor\frac{n}{2}\right\rfloor$ edges are assigned by 1 . Here, the sum of the weight of all the edges at every 
Edge Roman Star Domination Number on Graphs

vertex is either 2 or 1 and the edge of weight 0 has a neighboring edge with weigh 2 . Hence, $f$ is a $E R S D F$.

Thus, $\gamma_{R S}^{\prime}\left(C_{n}\right)=2\left|E_{2}\right|+\left|E_{1}\right|=2\left(\frac{1}{2}\left\lfloor\frac{n}{2}\right\rfloor\right)+\frac{1}{2}\left\lfloor\frac{n}{2}\right\rfloor=\frac{3}{2}\left\lfloor\frac{n}{2}\right\rfloor$

If the cycle is odd and $n=3$, then it is obvious, $\gamma_{R S}^{\prime}\left(C_{n}\right)=3$.

If the cycle is odd and $n>3$, then assign the edges as follows

$$
\begin{aligned}
f(e) & =0, e \in M \\
& =2, \text { edge joining two edges in } M \\
& =1, \text { otherwise. }
\end{aligned}
$$

Since the matching number is $\left\lfloor\frac{n}{2}\right\rfloor$, therefore, $\left|E_{0}\right|=\left\lfloor\frac{n}{2}\right\rfloor$. Since the edge with weight 2 is a link edge of the two edges with weight 0 , then $\left|E_{2}\right|=\left\lfloor\frac{n}{2}\right\rfloor-1$. Thus,

$$
\begin{gathered}
\left.\left|E_{1}\right|=|E|-\left(\left|E_{0}\right|+\left|E_{2}\right|\right)=n-\left(\left\lfloor\frac{n}{2}\right\rfloor+\left\lfloor\frac{n}{2}\right\rfloor-1\right]\right)=\left\lceil\frac{n}{2}\right\rfloor-\left\lfloor\frac{n}{2}\right\rfloor+1=1+1=2 \\
\text { Hence, } \gamma_{R S}^{\prime}\left(C_{n}\right)=2\left|E_{2}\right|+\left|E_{1}\right|=2\left(\left\lfloor\frac{n}{2}\right\rfloor-1\right)+2=2\left\lfloor\frac{n}{2}\right\rfloor .
\end{gathered}
$$

Lemma 4. Let $T$ be a complete binary tree, then $\gamma_{R S}^{\prime}(T)=2\left\lceil\frac{m-p}{3}\right\rceil+p$.

Proof: Let $T$ be a complete binary tree and $P$ be a set of $p$ number of pendant edges. Let $M$ be a matching of the induced subgraph $T \backslash P$.

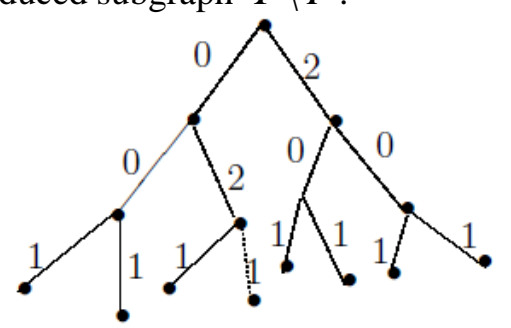

Figure 4: Tree

Assignment of the edges as follows,

$$
\begin{aligned}
f(e) & =2, e \in M \\
& =1, e \in P \\
& =0, \text { otherwise. }
\end{aligned}
$$

It is already proved that if the weight of each pendant edge is 1 , then $f$ is $E R S D F$. The number of edges in the matching $M$ of the set $E \backslash P$ is $\left\lceil\frac{m-p}{3}\right\rceil$. Assigning weight 2 to the edges in $M$ and weight 0 to the other remaining edges, it 


\section{Angshu Kumar Sinha, Akul Rana and Anita Pal}

observed that the sum of the weight of adjacent edges at each vertex is either one or two and each edge with weight 0 has at least one neighboring edge with 2 which ensure that $f$ is $E R S D F$.

Thus, $\gamma_{R S}^{\prime}(T)=2\left|E_{2}\right|+\left|E_{1}\right|=2\left\lceil\frac{m-p}{3}\right\rceil+p$.

\section{Some bounds}

In this section, we establish the boundary value of $\gamma_{R S}^{\prime}(G)$ of the general graphs $G$.

Lemma 5. For the graph $G$ with $n$ vertices and $m$ edges, $\frac{n}{2} \leq \gamma_{R S}^{\prime}(G) \leq m$.

Proof: Let $G$ be a graph of $n$ vertices and $m$ edges. let $f$ is a $E R S D F$, then $\sum_{e \in E\left(v_{i}\right)} f(e) \geq 1$ for every $v_{i} \in V, i=1,2,3, \ldots \ldots \ldots, n$.

Therefore, $\sum_{i=1}^{n} \sum_{e \in E\left(v_{i}\right)} f(e) \geq \sum_{i=1}^{n} 1=n$.

For every vertex $v_{i}, 2\left|E_{2}\left(v_{i}\right)\right|+\left|E_{1}\left(v_{i}\right)\right|+0\left|E_{0}\left(v_{i}\right)\right|=\sum_{e \in E\left(v_{i}\right)} f(e)$

Thus, $\sum_{i=1}^{n}\left(2\left|E_{2}\left(v_{i}\right)\right|+\left|E_{1}\left(v_{i}\right)\right|\right)=\sum_{i=1}^{n} \sum_{e \in E\left(v_{i}\right)} f(e)$

$$
\begin{aligned}
& 2 \sum_{i=1}^{n}\left|E_{2}\left(v_{i}\right)\right|+\sum_{i=1}^{n}\left|E_{1}\left(v_{i}\right)\right|=\sum_{i=1}^{n} \sum_{e \in E\left(v_{i}\right)} f(e) \\
& 2\left(\left|E_{2}\left(v_{1}\right)\right|+\left|E_{2}\left(v_{2}\right)\right|+\ldots .+\left|E_{2}\left(v_{n}\right)\right|\right)+\left|E_{1}\left(v_{1}\right)\right|+\left|E_{1}\left(v_{2}\right)\right|+\ldots+\left|E_{1}\left(v_{n}\right)\right|=\sum_{i=1}^{n} \sum_{e \in E\left(v_{i}\right)} f(e)
\end{aligned}
$$

Since every edge is incident with exactly two vertices, each edge gets counted twice, once at each end.

Therefore, $2\left(2\left|E_{2}\right|+\left|E_{1}\right|\right)=\sum_{i=1}^{n} \sum_{e \in E\left(v_{i}\right)} f(e)$

$$
\begin{aligned}
& 2\left|E_{2}\right|+\left|E_{1}\right|=\frac{1}{2} \sum_{i=1}^{n} \sum_{e \in E\left(v_{i}\right)} f(e) \\
& \gamma_{R S}^{\prime}(G)=\frac{1}{2} \sum_{i=1}^{n} \sum_{e \in E\left(v_{i}\right)} f(e) \geq \frac{n}{2}, \text { using (1). }
\end{aligned}
$$

Hence, $\gamma_{R S}^{\prime}(G) \geq \frac{n}{2}$.

Now if the assignment of each edge is 1 , then one can easily obtain the maximum value of $\gamma_{R S}^{\prime}(G)$.

Hence, $\gamma_{R S}^{\prime}(G) \leq m$

Combine the two results $\gamma_{R S}^{\prime}(G) \geq \frac{n}{2}$ and $\gamma_{R S}^{\prime}(G) \leq m$, we have $\frac{n}{2} \leq \gamma_{R S}^{\prime}(G) \leq m$. 
Edge Roman Star Domination Number on Graphs

Equality $\frac{n}{2} \leq \gamma_{R S}^{\prime}(G)$ holds for $C_{n}$ where $n$ is even.

The equality $\gamma_{R S}^{\prime}(G) \leq m$ holds for the path $P_{m}$ where $m \leq 5$. The upper bounds also hold for the graph $K_{1, n}$ and for the graph $K_{3}$.

Lemma 6. For the graph $G, 0 \leq \gamma_{R}^{\prime}(G) \leq \gamma_{R S}^{\prime}(G)$.

Proof: The proof is obvious.

Lemma 7. For any graph $G, \gamma^{\prime}(G) \leq \gamma_{R S}^{\prime}(G) \leq 2 \gamma^{\prime}(G)$.

Proof : Let $f$ be a $\gamma_{R S}^{\prime}(G)$ be a function. Then, $E_{1} \cup E_{2}$ is an edge dominating set of $G$. $\gamma^{\prime}(G)=\left|E_{1}\right|+\left|E_{2}\right| \leq\left|E_{1}\right|+2\left|E_{2}\right|=\gamma_{R S}^{\prime}(G)$.

Again, $2 \gamma^{\prime}(G)=2\left(\left|E_{1}\right|+\left|E_{2}\right|\right)=\left|E_{1}\right|+2\left|E_{2}\right|+\left|E_{1}\right|=\gamma_{R S}^{\prime}(G)+\left|E_{1}\right| \geq \gamma_{R S}^{\prime}(G)$.

Hence, $\gamma^{\prime}(G) \leq \gamma_{R S}^{\prime}(G) \leq 2 \gamma^{\prime}(G)$.

Lemma 8. For any graph $G, \gamma_{s r s}^{\prime}(G) \leq \gamma_{R S}^{\prime}(G)$.

Proof : For any graph $G$, let $f^{\prime}$ be a signed edge roman star domination function. Then, by definition of SERSD, $\gamma_{s r s}^{\prime}(G)=2\left|E_{2}\right|+\left|E_{1}\right|-\left|E_{-1}\right|$.

Now to modify $f^{\prime}$ to $f$ so that it's becoming an edge Roman domination function, the edges with weight -1 are changed to the weight 0 . After modification of $f^{\prime}$ to $f$, the set of edges in $E_{-1}$ becomes the set of edges in $E_{0}$.

Thus we have, $\gamma_{\text {srs }}^{\prime}(G)=2\left|E_{2}\right|+\left|E_{1}\right|-\left|E_{-1}\right| \leq 2 E_{2}+E_{1}=\gamma_{R S}^{\prime}(G)$, equality holds for $K_{2}$

\section{Conclusion}

In this paper, we introduce a new variation of edge Roman domination called edge Roman star domination.In this paper, we have found the exact value of edge roman star domination number of star graphs, paths, cycles, complete binary trees and Petersen graphs. Also, bounds of signed Roman star edge domination number are presented for general graphs in terms of the number of vertices and edges. Further study can be done to investigate ERSDN of the wheel, Bipartite, Petersen graphs and other graphs not studied here.

\section{REFERENCES}

1. S.Akbari, S.Bolouki, P.Hatami and M.Siami, On the signed edge domination number of graphs, Discrete Mathemaics, 309 (2009) 587-594.

2. H.A.Ahangar, M.A.Henning, C.L.Owenstein, Y.Zhao and V.Samodivkin, Signed Roman domination in graphs, J. Comb.Optim., 27 (2014) 241-255.

3. H.A.Ahangar, M.A.Henning, C.Löwenstein, Y.Zhao and V.Samodivkin, Signed Roman domination in graphs, J. Comb. Optim., 27 (2014) 241-255.

4. H.A.Ahangar, J.Amjadi, S.M.Sheikholeslami, L.Volkmann and Y.Zhao, Signed Roman edge domination numbers in graphs, J. Comb. Optim., 31 (2016) 333-346 
Angshu Kumar Sinha, Akul Rana and Anita Pal

5. S.Akbari, S.Ehsani, S.Ghajar, P. Jalaly Khalilabadi and S.Sadeghian Sadeghabad, On the edge Roman domination in graphs, manuscript.

6. J.G.Chang, Algorithmic aspects of domination in graphs, Handbook of Combinatorial Optimization (D.-Z. Du and P. M. Pardalos eds.), 3 (1998) 339-405.

7. G.J.Chang, S.-H.Chen and C.-H.Liu, Edge Roman domination on graphs, Graphs and Combinatorice, 32 (2016) 1731-1747

8. E.J.Cockayne and S.T.Hedetniemi, Towards a theory of domination in graphs. Networks, 7 (1977) 247-261.

9. E.J.Cockayne, P.A.Dreyer Jr, S.M.Hedetniemi and S.T.Hedetniemi, Roman domination in graphs, Discrete Math., 278 (2004) 11-22.

10. O.Favaron, H.Karami, R.Khoeilar and S.M.Sheikholeslami, On the Roman domination number of a graph, Discrete Math., 309 (2009) 3447-3451.

11. T.W.Haynes, S.T.Hedetniemi and P.J.Slater, Fundamentals of Domination in Graphs, Marcel Dekker Inc., New York (1998).

12. T.W.Haynes, S.T.Hedetniemi and P.J.Slater, Domination in Graphs: Advanced Topics, Marcel Dekker Inc., New York (1998).

13. S.Mitchell and S.T.Hedetniemi, Edge domination in trees, Congr. Numer., 19 (1977) 489-509.

14. O.Ore, Theory of Graphs, Amer. Math. Soc. Colloq. Publ., 38 (1962).

15. P.Roushini, L.Pushpam and T.N.M.Nalini Mai, Edge Roman domination in graphs, $J$. Combin. Math. Combin. Comput., 6 (2009) 175-182.

16. C.S.ReVelle and K.E.Rosing, Defendens imperium Roman: A classical problem in military strategy, Amer. Math. Monthly, 107 (2000) 585-594.

17. A.K.Sinha, A.Rana and A.Pal, The 2-tuple domination problem on trapezoid graphs, Annals of Pure and Applied Mathematics, 7 (2014) 71-76

18. S.M.Sheikholeslami and L.Volkmann, The signed Roman domatic number of a graph, Ann. Math. Inform., 40 (2012) 105-112.

19. S.M.Sheikholeslami and L.Volkmann, Signed Roman domination number of a digraph, J. Comb. Optim., 30 (2015) 456-467.

20. S.M.Sheikholeslami, S.M.L.Volkmann, The signed Roman domatic number of a digraph, Electron J Graph Theory Appl., 3 (2015) 85-93.

21. A.K.Sinha, A.Rana and A.Pal, Signed star domination number on proper interval graphs, International Journal of Mathematics, 106 (2016) 123-129.

22. A.K.Sinha, A.Rana and A.Pal, Signed edge domination number on interval graphs, communicated.

23. A.K.Sinha, A.Rana and A.Pal, Signed Roman edge star domination on graphs, Communicated.

24. I.Stewart, Defend the Roman Empire!, Sci. Amer., 281 (1999) 136-139.

25. B.Xu, On signed edge domination numbers of graphs, Discrete Mathematics, 239 (2001) 179-189.

26. B.Xu, On edge domination numbers of graphs, Discrete Mathematics, 294 (2005) 311-316. 